\title{
Immunogenicity of Exosomes from Dendritic Cells Stimulated with Toxoplasma gondii Lysates in Ocularly Immunized Mice
}

\author{
Bong-Kwang Jung ${ }^{1, \dagger}$, Eun-Do Kim ${ }^{2,3, \dagger}$, Hyemi Song ${ }^{1}$, Jong-Yil Chai ${ }^{1,4}$, Kyoung Yul Seo ${ }^{2, *}$ \\ ${ }^{1}$ Institute of Parasitic Diseases, Korea Association of Health Promotion, Seoul 07649, Korea; ${ }^{2}$ Department of Ophthalmology, Severance Eye \\ Hospital, Severance Hospital, Institute of Vision Research, Yonsei University College of Medicine, Seoul 03722, Korea; ${ }^{3}$ Mucosal Immunobiology \\ Section, Laboratory of Molecular Immunology, National Institute of Allergy and Infectious Diseases, NIH, Bethesda, Maryland, USA; ${ }^{4}$ Department of \\ Tropical Medicine and Parasitology, Seoul National University College of Medicine, Seoul 03080, Korea
}

\begin{abstract}
Immunogenicity of dendritic cell-derived exosomes stimulated with Toxoplasma gondii lysates (TLA exo), mixed with cholera toxin as an adjuvant, was investigated in mice immunized via 2 mucosal routes (ocular vs intranasal). BALB/c mice were injected 3 times with TLA exo vaccine at 2 week interval, and the levels of $\lg$ in serum and $\lg A$ in tear, saliva, feces, and vaginal wash were measured. To observe the expression of $T$. gondii-specific B1 gene, mice infected with ME49 T. gondii cysts were immunized with TLA exo or PBS exo (not stimulated with TLA), and their brain tissues were examined. The mice vaccinated via intranasal route elicited significantly higher humoral and mucosal immune responses compared with mice treated with PBS alone. Also, mice immunized via ocular route (by eyedrop) induced significantly higher T. gondiispecific lgG in serum and IgA in tear and feces in comparison with PBS controls. B1 gene expression was significantly lower in TLA exo vaccinated mice than in PBS or PBS exo vaccinated mice. These results demonstrated that ocular immunization of mice with TLA exo vaccine has the potential to stimulate systemic or local antibody responses. This study also highlighted an advantage of an eyedrop vaccine as an alternative for T. gondii intranasal vaccines.
\end{abstract}

Key words: Toxoplasma gondii, exosome, dendritic cell, mucosal vaccine, ocular vaccine

Toxoplasma gondii (T. gondii) is an obligate intracellular parasite that infects a wide range of hosts, including humans and domestic animals, throughout the world [1]. The infective forms of Toxoplasma initially invade the intestinal epithelium of the host, and then the parasite can lead to a congenital infection during pregnancy and also can lead to severe morbidity and mortality in patients suffering from immunocompromised conditions [2]. In veterinary industries, T. gondii infection is economically important since it can cause abortion and neonatal loss of sheep and goats [3]. Thus, the effective vaccine against Toxoplasma would be important for both humans and veterinary medical fields.

Mucosal vaccines, including nasal, oral, vaginal, and sublingual ones, have been developed during the last decades [4].

- Received 20 December 2019, revised 23 March 2020, accepted 23 March 2020

*Corresponding author (seoky@yuhs.ac)

†These authors equally contributed to this work.

(C) 2020, Korean Society for Parasitology and Tropical Medicine

This is an Open Access article distributed under the terms of the Creative Commons Attribution Non-Commercial License (https://creativecommons.org/licenses/by-nc/4.0) which permits unrestricted non-commercial use, distribution, and reproduction in any

medium, provided the original work is properly cited.
Several oral and intranasal vaccines against cholera, polio, and influenza have been commercially available since 2003, and a commercial intranasal influenza vaccine for humans (Flumist ${ }^{\circledR}$ ) began to be used [5]. However, this vaccine was forbidden by Centers for Disease Control and Prevention (CDC) in USA after 2017 because of its inefficient protection against influenza among 2-17 year-old children [6]. Moreover, it could provoke narcolepsy and has been prohibited for use by the Korean Government.

Recently, eyedrop vaccines have been studied as an alternative mucosal vaccine, in various animal models, including fowls, bovines, mice, and ferrets, and proved to be effective for protecting hosts from pathogens $[7,8]$. Unlike intranasal vaccines, no ocularly inoculated materials enter the central nervous system (CNS), and ocular vaccines have been reported to have no side effects on CNS [7,9]. However, there have been few studies evaluating the effects of eyedrop vaccines against $T$. gondii or other kinds of parasitic infections.

Recently, new vaccines using parasite-derived extracellular vesicles (EV), which contain exosomes and interact with host 
immunity, were reported [10-12]. In particular, dendritic cells (DCs) are known to secrete exosomes expressing functional MHC class I/II and T-cell costimulatory molecules on the surface of EV [13]. The properties of these structures are compatible for their use as an alternative vaccine to DC-based cell-free vaccines [13]. In the present study, we evaluated the immunogenicity of a Toxoplasma-DC-derived exosome vaccine inoculated via eye mucosa in comparison with an intranasal vaccine for inducing protective immunity against $T$. gondii in mice.

DC2.4 cells, a mouse DC line, were cultured in Dulbecco's Modified Eagle's Medium (DMEM, Welgene, Seoul, Korea) supplemented with 10\% fetal bovine serum (FBS; Welgene), 4 $\mathrm{mM}$ L-glutamine, $0.2 \mathrm{mM}$ penicillin, and $0.05 \mathrm{mM}$ streptomycin (Welgene) at $37^{\circ} \mathrm{C}$ in a $5 \% \mathrm{CO}_{2}$ incubator. Toxoplasma lysate antigen (TLA) was prepared as previously described with slight modifications [14]. Parasites were resuspended in PBS (pH 7.4) and sonicated on ice. The supernatant, i.e., TLA, was filter-sterilized through a $0.22 \mu \mathrm{m}$ membrane, and the protein concentration was determined using a NanoDrop 2000 spectrophotometer (Thermo Scientific, Rockford, Illinois, USA). TLA was stored at $-70^{\circ} \mathrm{C}$ until required. T. gondii, ME49 strain, was maintained by injecting the cysts into the peritoneal cavity of $\mathrm{BALB} / \mathrm{C}$ mice (Orient Bio Animal Center, Seongnam, Korea). The ME49 cysts in the brain of mice were harvested 3 months after the infection by mincing the brain tissue. To each experimental mouse,

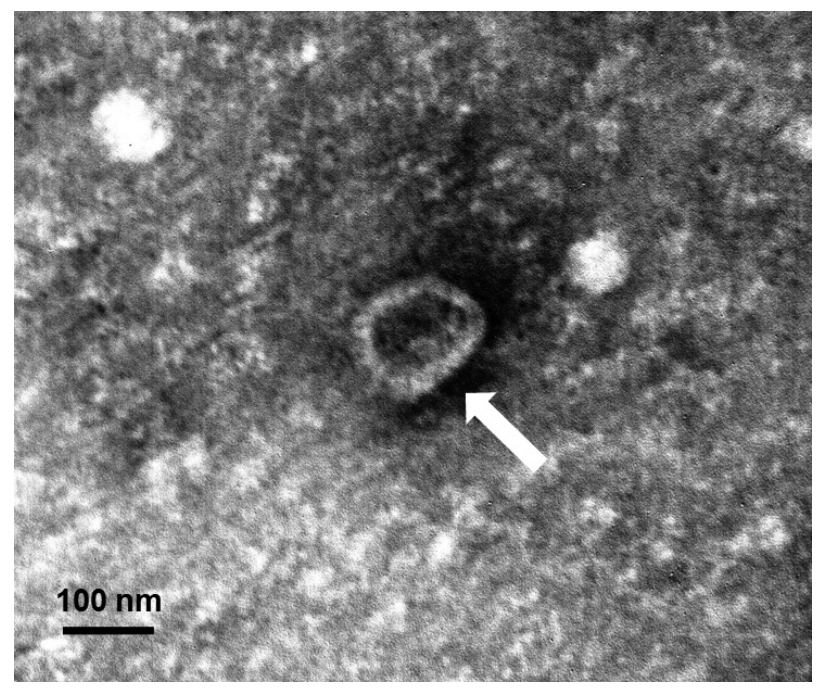

Fig. 1. A dendritic cell-derived exosome isolated by differential ultracentrifugation and negatively stained with $2 \%$ uranyl acetate. The observed exosome is shown as approximately $60-100 \mathrm{~nm}$ in size and cup-shaped in its structure under a transmission electron microscopy. A white arrow points an exosome.
30 cysts were inoculated intraperitoneally.

Exosomes from DC2.4 cells, stimulated with TLA (TLA exo) or not (PBS exo), were isolated by differential ultracentrifugation following the protocol described by Aline et al. [2]. The concentration of isolated exosomes was determined by BCA protein assay kit (Pierce, Rockford, Illinois, USA) and NanoDrop 2000 spectrophotometer. To observe the morphological characteristics, exosomes isolated from DC2.4 cells were negatively stained with $2 \%$ uranyl acetate for $1 \mathrm{~min}$ as previously described [15]. They were observed using a JEOL 1200-EX II transmission electron microscope (JEOL, Tokyo, Japan) equipped with an ES1000W Erlangshen CCD camera (Gatan, Pleasanton, California, USA) at an accelerating voltage of $80 \mathrm{kV}$. The exosomes were approximately $60-100 \mathrm{~nm}$ in size and cup-shaped (Fig. 1).

Specific pathogen-free BALB/c mice, aged 6-10 weeks, were purchased from Charles River Laboratories (Orient Bio, Sungnam, Korea). All mice were maintained in an experimental animal facility under specific pathogen-free conditions at the Department of Laboratory Animal Resources, Yonsei Biomedical Research Institute, Yonsei University (Seoul, Korea) and received sterilized food (Certified Diet MF; Oriental Yeast, Osa$\mathrm{ka}$, Japan) and filtered tap water. All animal experiments were approved by the Animal Research Committee of Yonsei University (IACUC 2015-0022, IBC 2014-034).

Prior to experimental manipulation, mice were anesthetized by intraperitoneal injection of ketamine $(100 \mathrm{mg} / \mathrm{kg}$ ) and xylazine hydrochloride $(10 \mathrm{mg} / \mathrm{kg})$. For intranasal or ocular immunization, $1 \mu \mathrm{g}$ of TLA or TLA exo Ags with $2 \mu \mathrm{g}$ cholera toxin (CT) (List Biological Laboratories, Campbell, California, USA) per head was suspended in $10 \mu \mathrm{l}$ PBS and dropped 3 times at 2 week interval onto the surface of the cornea making a droplet or intranasally onto the nostrils using a micropipette. For eye drop inoculation, $5 \mu \mathrm{l}$ was dropped per eye. To keep the vaccine solution as droplets on the eye and not overflown, the immunized mice were kept lying on mice's abdomen in the cage until they wake up. At week 2 after the last immunization, sera were obtained by retro-orbital bleeding. Tear wash samples were obtained by washing with $100 \mu$ PBS per eye Saliva was obtained following intraperitoneal injection of mice with pilocarpine (500 mg/kg; Sigma-Aldrich). Fecal samples were obtained by adding weighed feces to PBS containing $0.1 \%$ sodium azide. The feces were mixed by vortexing and centrifugation, and the supernatants were collected for assay. Vaginal wash samples were collected by lavage with $100 \mu \mathrm{l}$ 
PBS. After the mice were sacrificed, nasal wash samples were obtained by flushing $100 \mu \mathrm{l}$ PBS through the anterior (oral) entrance of the nasal passages using a pipette.

ELISA plates (Falcon, Franklin Lakes, New Jersey, USA) were coated with TLA $(1 \mathrm{mg} / \mathrm{ml})$ in PBS and were incubated overnight at $4^{\circ} \mathrm{C}$. Blocking was done with $1 \%$ BSA (Sigma-Aldrich) in PBS. Horseradish peroxidase (HRP)-conjugated goat antimouse IgG or IgA (Southern Biotechnology Associates, Birmingham, Alabama, USA) was added to each well and incubated overnight at $4^{\circ} \mathrm{C}$. For color development, tetramethylbenzidine solution (Moss, Pasadena, Maryland, USA) was used. Then, the plates were measured at $450 \mathrm{~nm}$ on an ELISA reader (Molecular Devices, Sunnyvale, California, USA) after addition of stopping solution $(0.5 \mathrm{~N} \mathrm{HCl})$. Endpoint titers of antigen-specific antibodies were expressed as reciprocal $\log _{2}$ titers of the last dilution that showed 0.1 absorbance over background levels.

Mice challenged with 30 ME49 cysts were observed for expression of T. gondii-specific gene (B1 gene) in the brain. At week 4 after the challenge, mice were killed and their brains were removed. Each brain was homogenized in $2 \mathrm{ml}$ of PBS with a mortar and pestle. Total genomic DNA was isolated from each brain homogenate using DNeasy blood and tissue kit (Qiagen, Hilden, Germany). The expression levels of T. gondii specific B1 gene were evaluated using real-time PCR. The $\mathrm{Ct}$ values were analyzed using the comparative $\mathrm{Ct}(\Delta \Delta \mathrm{Ct})$ method. Normalization was performed by using the reference gene $\beta$-actin relative to the control. Data are expressed as the mean $\pm S D$, and statistical analyses were done by the $t$-test (Excel program).

A

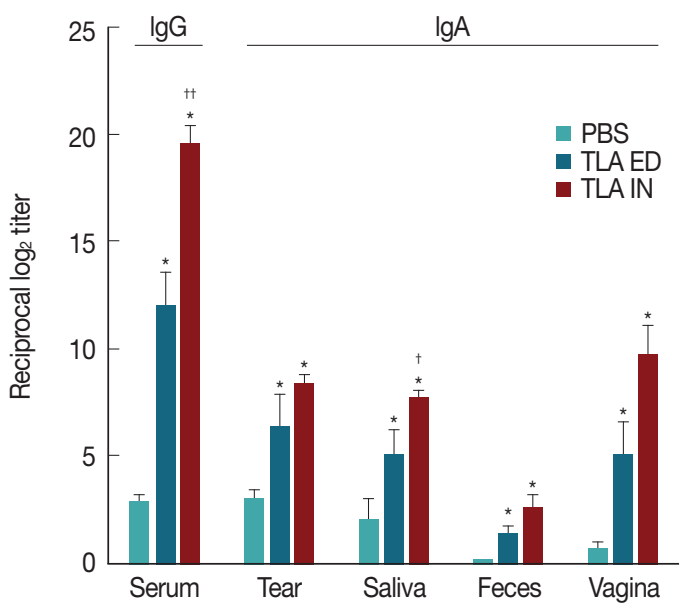

At week 2 after the third vaccination of TLA or TLA stimulated DC exosomes (TLA exo), the levels of TLA-specific serum IgG and mucosal IgA derived from tear, saliva, feces, and vagina were significantly increased in both eyedrop and intranasal vaccination groups (Fig. 2A). When TLA exo antigens derived from TLA-stimulated DCs were used, the increases of specific IgA antibody levels were higher in ED group than in IN group except in the saliva sample (Fig. 2B). However, TLA exo-specific serum IgG level was higher in IN group than in ED administration group (Fig. 2B). Although TLA exo-specific serum IgG levels were significantly increased at week 6 post-immunization in both ED and IN groups, IN vaccination did not induce significant increases of mucosal IgA except for the tear and feces samples (Fig. 2B). It was also shown that TLA exo protected $\mathrm{BALB} / \mathrm{c}$ mice against intraperitoneal challenge with $30 \mathrm{ME} 49$ cysts of $T$. gondii. The mice vaccinated with TLA exo and infected with ME49 cysts had body weights similar to those of the non-vaccinated infected group (data not shown).

At week 6 post-inoculation, we evaluated the expression of B1 gene as Toxoplasma-specific marker in the brain of TLA exo vaccinated mice (Fig. 3). The expression level of B1 gene was significantly lower in TLA exo vaccinated group than in PBS or PBS exo vaccinated mice. These results strongly suggested that the eyedrop TLA exo vaccination can help to reduce T. gondii specific gene in the brain of mice against acute $T$. gondii infection.

Our results demonstrated that the eyedrop administration of DC exosomes stimulated with TLA exhibited strong immunogenicity inducing immune responses in mice through the

B

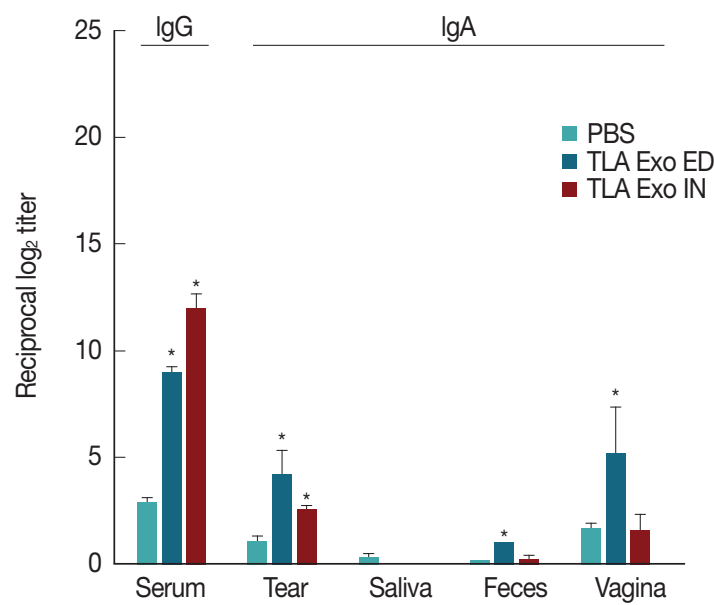

Fig. 2. Toxoplasma gondii-specific antibodies (IgG and $\operatorname{lgA}$ ) in serum and mucosal fluids of mice immunized through eyedrop (ED) or intranasal (IN) administration with T. gondii lysate antigens (TLA) in cholera toxin as an adjuvant (A) or with TLA stimulated dendritic cell-derived exosomes (TLA exo) in CT as an adjuvant (B). 


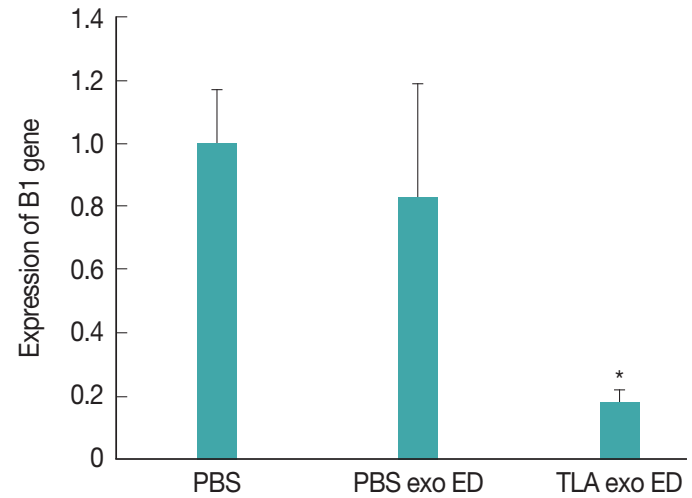

Fig. 3. Expression levels of Toxoplasma gondii-specific B1 gene in the brain of mice inoculated with TLA-stimulated dendritic cell-derived exosomes (TLA exo) using eyedrop (ED) vaccine administration.

production of T. gondii-specific antibodies and reduction of B1 gene expression against infection with ME49 cysts. This is the first report of anti-Toxoplasma effects of DC-derived exosomes vaccinated via mucosal route, such as eyedrop or intranasal inoculation.

Although eyedrop vaccination of TLA exo in mice induced lower levels of antigen-specific serum IgG or mucosal IgA than those of TLA, the levels of TLA exo-specific antibodies were significantly higher than those of PBS-treated controls. Mucosal samples from tear and feces in TLA exo treated mice showed significant increases of specific mucosal IgA and serum IgG levels. This may indicate that Toxoplasma antigens expressed or inserted on the exosomes derived from TLA-pulsed DCs was properly processed in DCs after the vaccine antigens were administered by eyedrop, so that successful Toxoplasma-specific immunity could be induced. We demonstrated that TLA exo could induce protective immunity in mice against the acute stage of T. gondii infection. BALB/C mice challenged with a sublethal dose of Toxoplasma cysts exhibited a dramatically lower expression of Toxoplasma B1 gene following parasite infection than that of untreated mice (PBS) or mice treated with exosomes derived from unpulsed DC2.4 cells (PBS exo). It is surmised that the eyedrop TLA exo vaccine could activate antigenspecific immune cells which lead to reduce the expression of B1 gene and inhibit the proliferation of Toxoplasma in the brain of infected mice.

The application scopes of EV, including exosomes and microvesicles (MV), is gradually expanding in the diagnostic and vaccination fields [16]. Especially, new approaches for vaccination were required to overcome the challenges for various infectious diseases [16]. Candidates of exosome vaccines have been suggested for various viruses, including Ebola virus, influenza virus, Crimean-Congo hemorrhagic fever virus, West Nile virus, and hepatitis $\mathrm{C}$ virus, and parasites, such as Trichuris, Leishmania, Schistosoma, and Toxoplasma [16-20].

Some groups evaluated the potential utility of DC-derived exosomes to mediate protective immunity against cutaneous leishmaniasis [18]. These findings suggested that the development of a cell-free vaccine for immunoprophylaxis against leishmaniasis and other infectious diseases is feasible [18]. Another group reported that $\mathrm{EV}$ proteomic analysis showed numerous known vaccine candidates against schistosomes [19]. The analysis provided new avenues for the exploration of host-schistosome interactions and offered a potential mechanism by which some vaccine antigens exert their protective efficacy [19]. EVs isolated from Trichuris muris also induced protective immunity in mice when administered as a vaccine without an adjuvant [20]. Several reports suggested innovative methods for discovery and development of new therapeutic strategies [21].

Normally, the contents of exosomes depend on the state (after external stimulation) of the stimulating cells [12]. In this study, we speculate that there may be specific antigens of Toxoplasma in exosomes derived from DCs stimulated with TLA through the production of T. gondii specific antibodies (Fig. 2). However, further studies are needed to explore the contents of exosomes derived from DCs stimulated with T. gondii through genomic and proteomic analyses.

The present study has a limitation in that it confirmed only the efficacy of eye-drop exosome vaccines rather than evaluating the safety of the vaccines. For developing safe vaccine candidates, we need to approach with various strategies, including use of alternative and safe adjuvants rather than CT. Further studies should be focused on new approaches using alternative adjuvant candidates for developing safe vaccines.

In conclusion, our results report a new approach of TLAstimulated DC-derived exosomes for vaccination against Toxoplasma infection. Further studies are needed to identify several antigens within exosomes which can be targeted by host antibodies following vaccination. Although eyedrop vaccination needs to be evaluated for its further usefulness and limitations for clinical trials, it has the potential of an easy and alternative method for inducing protective immune responses against Toxoplasma infection. 


\section{ACKNOWLEDGMENTS}

This research was supported by Korea Mouse Phenotyping Project (NRF-2013M3A9D5072551) from the Ministry of Science and ICT through the National Research Foundation.

\section{CONFLICT OF INTEREST}

We have no conflict of interest related to this work.

\section{REFERENCES}

1. Tenter AM, Heckeroth AR, Weiss LM. Toxoplasma gondii: from animals to humans. Int J Parasitol 2000; 30: 1217-1258.

2. Aline F, Bout D, Amigorena S, Roingeard P, Dimier-Poisson I. Toxoplasma gondii antigen-pulsed-dendritic cell-derived exosomes induce a protective immune response against $T$. gondii infection. Infect Immun 2004; 72: 4127-4137.

3. Dubey JP. Toxoplasmosis in sheep-the last 20 years. Vet Parasitol 2009; 163: 1-14.

4. Holmgren J, Czerkinsky C. Mucosal immunity and vaccines. Nat Med 2005; 11(suppl): 45-53.

5. Zheng Z, Diaz-Arévalo D, Guan H, Zeng M. Noninvasive vaccination against infectious diseases. Hum Vaccin Immunother 2018; 14: 1717-1733.

6. Chung JR, Flannery B, Ambrose CS, Bégué RE, Caspard H, DeMarcus L, Fowlkes AL, Kersellius G, Steffens A, Fry AM. Live attenuated and inactivated influenza vaccine effectiveness. Pediatrics 2019; 143: e20182094

7. Seo KY, Han SJ, Cha HR, Seo SU, Song JH, Chung SH, Kweon $\mathrm{MN}$. Eye mucosa: an efficient vaccine delivery route for inducing protective immunity. J Immunol 2010; 185: 3610-3619.

8. Yoon S, Kim ED, Song MS, Han SJ, Park TK, Choi KS, Choi YK, Seo KY. Eyedrop vaccination induced systemic and mucosal immunity against influenza virus in ferrets. PLoS One 2016; 11: e0157634.

9. Kim ED, Han SJ, Byun YH, Yoon SC, Choi KS, Seong BL, Seo KY. Inactivated eyedrop influenza vaccine adjuvanted with poly (I:C) is safe and effective for inducing protective systemic and mucosal immunity. PLoS One 2015; 10: e0137608.

10. Silverman JM, Clos J, Horakova E, Wang AY, Wiesgigl M, Kelly I, Lynn MA, McMaster WR, Foster LJ, Levings MK, Reiner NE. Leishmania exosomes modulate innate and adaptive immune re- sponses through effects on monocytes and dendritic cells. J Immunol 2010; 185: 5011-5022.

11. Zamanian M, Fraser LM, Agbedanu PN, Harischandra H, Moorhead AR, Day TA, Bartholomay LC, Kimber MJ. Release of small RNA-containing exosome-like vesicles from the human filarial parasite Brugia malayi. PLoS Negl Trop Dis 2015; 9: e0004069.

12. Buck AH, Coakley G, Simbari F, McSorley HJ, Quintana JF, Le Bihan T, Kumar S, Abreu-Goodger C, Lear M, Harcus Y, Ceroni A, Babayan SA, Blaxter M, Ivens A, Maizels RM. Exosomes secreted by nematode parasites transfer small RNAs to mammalian cells and modulate innate immunity. Nat Commun 2014; 5: 5488.

13. Keller S, Sanderson MP, Stoeck A, Altevogt P. Exosomes: from biogenesis and secretion to biological function. Immunol Lett 2006; 107: 102-108.

14. Jung BK, Pyo KH, Shin K, Hwang Y, Lim H, Lee S, Moon JH, Lee S, Suh YH, Chai JY, Shin EH. Toxoplasma gondii infection in the brain inhibits neuronal degeneration and learning and memory impairments in a murine model of Alzheimer's disease. PLoS One 2012; 7: e33312.

15. Perez-Hernandez J, Forner MJ, Pinto C, Chaves F, Cortes R, Redon J. Increased urinary exosomal microRNAs in patients with systemic lupus erythematosus. PLoS One 2015; 10: e0138618.

16. Jungbauer A. Exosomes enter vaccine development: strategies meeting global challenges of emerging infections. Biotechnol J 2018; 13: e1700749.

17. Anticoli S, Manfredi F, Chiozzini C, Arenaccio C, Olivetta E, Ferrantelli F, Capocefalo A, Falcone E, Ruggieri A, Federico M. An exosome-based vaccine platform imparts cytotoxic T lymphocyte immunity against viral antigens. Biotechnol J 2018; 13: e1700443.

18. Schnitzer JK, Berzel S, Fajardo-Moser M, Remer KA, Moll H. Fragments of antigen-loaded dendritic cells (DC) and DC-derived exosomes induce protective immunity against Leishmania major. Vaccine 2010; 28: 5785-5793.

19. Sotillo J, Pearson M, Potriquet J, Becker L, Pickering D, Mulvenna J, Loukas A. Extracellular vesicles secreted by Schistosoma mansoni contain protein vaccine candidates. Int J Parasitol 2016; 46: $1-5$.

20. Shears RK, Bancroft AJ, Hughes GW, Grencis RK, Thornton DJ. Extracellular vesicles induce protective immunity against Trichuris muris. Parasite Immunol 2018; 40: e12536.

21. Eichenberger RM, Sotillo J, Loukas A. Immunobiology of parasitic worm extracellular vesicles. Immunol Cell Biol 2018; 96: 704-713. 
